\title{
Calcitonin Gene Related Peptide A Is Dispensable for Many Danger-related Motivational Responses
}

\author{
Joanna Zajdel \\ Linköping University \\ Johan Sköld \\ Linköping University \\ Maarit Jaarola \\ Linköping University \\ Anand Kumar Singh \\ Linköping University \\ David Engblom ( $\sim$ david.engblom@liu.se ) \\ Linköping University
}

\section{Research Article}

Keywords: Calcitonin-gene related peptide alpha, parabrachial-amygdaloid pathway, nociception, anorexia, aversive learning, conditioned taste avoidance

Posted Date: February 23rd, 2021

DOI: https://doi.org/10.21203/rs.3.rs-236137/v1

License: (c) (i) This work is licensed under a Creative Commons Attribution 4.0 International License. Read Full License

Version of Record: A version of this preprint was published at Scientific Reports on August 10th, 2021. See the published version at https://doi.org/10.1038/s41598-021-95670-8. 


\title{
Calcitonin gene related peptide $\alpha$ is dispensable for many danger-related motivational responses
}

\author{
Joanna Zajdel ${ }^{1,2}$, Johan Sköld ${ }^{1,2}$, Maarit Jaarola ${ }^{1}$, Anand Kumar Singh ${ }^{1}$ and David Engblom ${ }^{1 *}$ \\ ${ }^{1}$ Center of Social and Affective Neuroscience, Department of Biomedical and Clinical Sciences, \\ Linköping University, 58185 Linköping, Sweden \\ ${ }^{2}$ These authors contributed equally \\ *Corresponding author: david.engblom@liu.se
}

\begin{abstract}
Calcitonin gene related peptide (CGRP) expressing neurons in the parabrachial nucleus have been shown to encode danger. Through projections to the amygdala and other forebrain structures, they regulate food intake and trigger adaptive behaviors in response to threats like inflammation, intoxication, tumors and pain. Despite the fact that this danger-encoding neuronal population has been defined based on its CGRP expression, it is not clear if CGRP is critical for its function. To examine the role of CGRP in danger-related motivational responses, we used male and female mice lacking aCGRP, which is the main form of CGRP in the brain. These mice had no, or only very weak, CGRP expression in the parabrachial nucleus and its projections to the amygdala. Despite this, they displayed normal danger-related responses such as inflammation-induced anorexia and conditioned taste aversion. Further, mice lacking aCGRP showed normal nociceptive responses, intact aversion to thermal pain and close to normal pain-induced escape behavior. Collectively, our findings suggest that aCGRP is not necessary for short term danger-encoding and threat-related responses but that the parabrachial CGRP expressing neurons use other transmitters for these functions.
\end{abstract}

\section{Keywords}

Calcitonin-gene related peptide alpha, parabrachial-amygdaloid pathway, nociception, anorexia, aversive learning, conditioned taste avoidance 


\section{Introduction}

Calcitonin gene related peptide-expressing neurons in the external lateral part of the parabrachial nucleus (CGRP ${ }^{\mathrm{PBN}}$ neurons) have been proposed to serve as a universal danger sensor. They are activated by a wide variety of noxious stimuli ${ }^{1,2}$, ranging from painful electric shocks, pinching and heat pain ${ }^{1,3}$, to malaise-inducing LiCl, lipopolysaccharide (LPS), satiety hormones ${ }^{4,5}$ and malignancies ${ }^{6}$. Activation of CGRPPBN neurons enables appropriate behavioral reactions to danger, such as suppression of appetite in conditions when food consumption might be harmful ${ }^{4,7}$, scratching in response to itch ${ }^{1}$ and the generation of threat memories ${ }^{3}$.

CGRP $^{\mathrm{PBN}}$ neurons send projections to the central amygdala (CeA) and other forebrain structures ${ }^{8}$. The projection to the extended amygdala have been shown to be important for several pain-related responses 3 . While the role of CGRP ${ }^{\text {PBN }}$ neurons is well established, less is known about the involvement of CGRP in the processes they mediate. Injections of CGRP to the amygdala have been reported to facilitate both synaptic transmission and pain responses ${ }^{10}$, but also to have an antinociceptive effect ${ }^{11}$, while infusions of CGRP receptor 1 antagonists have been demonstrated to block synaptic plasticity accompanying the development of chronic pain in rat model of arthritis ${ }^{12}$. Further, CGRP has been shown to modulate conditioned taste aversion induced by LiCl but not aversion induced by activation of CGRP ${ }^{\mathrm{PBN}}$ neurons ${ }^{13}$.

To address the role of $\alpha$ CGRP in functions mediated by CGRP ${ }^{\mathrm{PBN}}$ neurons, we used a transgenic mice with deletion of $\mathrm{Calca}^{14}$, the gene encoding $\alpha$ CGRP, which is the primary form of CGRP in the brain. In these mice, we investigated a broad panel of threat-related responses and behaviors including food intake suppression and pain-related responses.

\section{Materials and Methods}

\section{Animals}

All experiments were approved by the Linköping Animal Care and Use Committee and followed national and international guidelines including ARRIVE. Unless otherwise specified, mice were kept on a $12 \mathrm{~h}$ light/dark cycle with ad libitum access to food and water, in a room with controlled humidity and temperature. Pain, palatable food intake and c-fos induction experiments were performed during the light phase while conditioned taste aversion and anorexia experiments were performed during the dark phase. Animals were of both sexes and at least 7 weeks old at the start of the experiments. Wildtype mice used in the c-fos induction experiment were bred inhouse. $\alpha$ CGRP-KO mice ${ }^{14}$ were obtained from MMRRC and maintained on C57BL/6 background. The transgenic line was originally generated for ablation of Calca expressing cells ${ }^{14}$, but without administration of diphtheria toxin it can 
be used as a Calca-KO. Mice homozygous for the transgene are referred to as $\alpha \mathrm{CGRP}-\mathrm{KO}$, heterozygous mice as $\mathrm{HZ}$ and mice without the transgene as WT.

\section{C-fos induction and immunofluorescence}

Mice were perfused with saline followed by $4 \%$ paraformaldehyde in PBS. The brains were postfixed in the same solution for $4 \mathrm{~h}$ in $4^{\circ} \mathrm{C}$ and placed in $30 \%$ sucrose till saturated. For c-fos induction experiments, mice were injected with LPS (10 $\mu \mathrm{g} / \mathrm{kg}$, E. coli, 055:B5, Sigma, dissolved in sterile saline) or saline $3 \mathrm{~h}$ before perfusion. $40 \mu \mathrm{m}$ sections were cut in a cryostat and later blocked in $10 \%$ Normal Donkey Serum in PBST (PBS and 0.4\% Triton-X) for 24h. After blocking, sections were incubated in a mixture of primary antibodies (rabbit anti-CGRP, 1: 5 000, PenLab, T-4031 or rabbit anti-C-fos, 1:1000, Abcam, 190289; chicken anti-GFP, 1:10 000, Abcam, ab13970) over night and later in mixture of secondary antibodies (goat anti-chicken Alexa Fluor 488, 1:1000 and donkey anti-rabbit Alexa Fluor 568, 1:1000 (CGRP staining) or 1:200 (c-fos staining)) for $2 \mathrm{~h}$. For double staining of CGRP and c-fos, sections were blocked for 45 minutes in $4 \%$ bovine serum albumin and $0.2 \%$ Triton- $\mathrm{X}$, incubated in rabbit anti-C-fos overnight followed by $2 \mathrm{~h}$ of incubation in donkey anti-rabbit Alexa Fluor 568 . They were then blocked in 10\% normal donkey serum in PBST and incubated in rabbit anti-CGRP overnight followed by $2 \mathrm{~h}$ incubation in donkey anti-rabbit Alexa Fluor 488, 1:1000. For c-fos quantification, the external lateral part of the PBN was identified with the help of CGRP/GFP immunoreactivity. Pictures of the PBN were taken with a Leica DMi8 fluorescent microscope. Images were processed with $\mathrm{Fiji}^{15}$ by a researcher blinded to the treatment. Background was subtracted using the built-in rolling ball algorithm. A threshold was set and the number of c-fos positive cells in the PBN were counted with the "Analyze particles" function. All other images were taken with a Zeiss LSM700 upright confocal microscope.

\section{LPS-induced anorexia}

Mice were single-housed five days before the start of the experiment. One hour before the onset of the dark phase, animals were injected with saline or LPS i.p. $(10 \mu \mathrm{g} / \mathrm{kg}, \mathrm{E}$. coli, O111:B4, Sigma, dissolved in sterile saline) and chow was withdrawn. At the onset of the dark phase, the mice got access to food and the intake was measured 3 and 6 hours later.

\section{Conditioned taste aversion induced by LPS}

Mice were single housed and moved to a room with inverted light cycle (lights on at 11 pm) 2 weeks before the experiment. Animals were habituated to $4 \mathrm{~h}$ water deprivation (from the onset of dark phase) for 4 days. On the conditioning day, following $4 \mathrm{~h}$ water deprivation, mice got access to $0.15 \%$ $(\mathrm{w} / \mathrm{v})$ saccharin in tap water. After $1 \mathrm{~h}$, mice received LPS i.p. $(10 \mu \mathrm{g} / \mathrm{kg}, \mathrm{E}$. coli, 0111:B4, Sigma, dissolved in saline) or saline, and the baseline intake of saccharin was measured. During the next two 
days, animals were exposed to $4 \mathrm{~h}$ water deprivation. The third day after LPS treatment, following $4 \mathrm{~h}$ water deprivation, animals got access to saccharine solution for $1 \mathrm{~h}$ and the intake was measured. Mice drinking less than $0.5 \mathrm{~g}$ of saccharine solution during the conditioning day were excluded, since in our experience consuming less than this amount is not enough to elicit reliable taste avoidance. The results shown come from two separate cohorts.

\section{Palatable food intake}

Mice were single-housed 5 days before the start of the experiment. Consumption of Fresubin Original drink, chocolate flavour, was measured for $2 \mathrm{~h}$, after a pre-exposition to the flavour.

\section{Formalin test}

Animals were placed in a transparent plexiglass box 20 (w) $\times 15$ (d) $\times 25$ (h) cm for $30 \mathrm{~min}$. After the acclimatization period, $20 \mu \mathrm{l}$ of diluted formalin (2.5\%) was injected below the skin on dorsal side of the right hindpaw. The behavior was recorded for $60 \mathrm{~min}$. Time spent performing nociceptive behaviors (paw licking, biting and shaking) was measured and expressed as nociceptive score for each 5 minute slot.

\section{Conditioned place aversion induced by thermal pain}

Male mice were single-housed at least one day before start of experiment. An Incremental Hot/Cold Plate Analgesia Meter (IITC Inc Life Science) was modified to serve as a conditioned place preference box with two chambers. On day 1 (pre-test), animals explored the apparatus for $15 \mathrm{~min}$. If the time spent in one chamber exceeded $12 \mathrm{~min}$, the mouse was excluded. On days 2-5 animals were placed in a non-preferred chamber with the temperature set to $30^{\circ} \mathrm{C}$, and in the preferred chamber with the temperature set to $45^{\circ} \mathrm{C}$. The conditioning sessions lasted $10 \mathrm{~min}$ or until the mouse jumped three times. They were separated by at least $4 \mathrm{~h}$ and the order of the sessions was altered daily. On day 6 , post-test was performed the same way as the pre-test. The aversion scores were calculated as the difference between the times spent in the pain-paired chamber in the post-test minus the times spent in the same chamber in the pre-test.

\section{Heat escape test}

Mice were single-housed at least one day before start of experiment. A $12 \mathrm{~cm}$ high platform was placed inside a plexiglass enclosure on an Incremental Hot/Cold Plate Analgesia Meter (IITC Inc Life Science). The floor temperature was set to $45^{\circ} \mathrm{C}$. The animals were placed on the hot floor and the time until they jumped, or tried to jump, up onto the platform was measured. Only male mice were included in the experiment since, according to our experience, not all female mice escape from a $45^{\circ} \mathrm{C}$ hot floor to a $12 \mathrm{~cm}$ high platform within the 10 -minute test period approved by the animal care committee, leading to high variability within the groups. 


\section{Acetic acid locomotion}

On day 1 , mice previously tested in the heat escape test were habituated to an open field box $(44 \mathrm{x}$ $44 \mathrm{~cm}$ ). After $20 \mathrm{~min}$ they were injected with $100 \mu \mathrm{l}$ saline intraperitoneally. The same procedure was repeated on day 2 and the session was recorded. On day 3 the saline was replaced with $0.6 \%$ acetic acid dissolved in saline. Videos were analyzed with Ethovision XT tracking software (Noldus) to measure locomotion. Reduction in locomotion was calculated as distance moved after saline injection subtracted from distance moved after acetic acid injection.

\section{Statistical analysis}

Data were analyzed with GraphPad Prism. Results are presented as mean \pm SEM. Comparisons of two groups were done using Student's T-test and comparisons involving more than two groups were performed using one-way or two-way ANOVA followed by Holm-Sidak's multiple comparisons test.

\section{Results}

Loss of CGRP in the projections to the central amygdala in aCGRP-KO mice

To confirm the deletion of CGRP in the parabrachial-amygdaloid pathway, we compared the CGRP expression in $\alpha$ CGRP-KO animals carrying two copies of the transgene (farnesylated eGFP knocked-in to the Calca locus) to that of heterozygous mice (carrying one copy of the transgene and one intact Calca allele). In both genotypes, GFP was found in the cell membranes of PBN neurons and their projections to the amygdala. GFP-immunoreactivity was used to facilitate identification of these structures in the aCGRP-KO mice. We found only very weak CGRP-immunoreactivity in the PBN of aCGRP-KO mice whereas strong CGRP labeling was seen in heterozygous mice (fig. 1A-F). Furthermore, no CGRP immunorectivity was detected in the CeA of aCGRP-KO mice (fig. 1G-L), indicating loss of CGRP signaling in this area.

\section{A low dose of lipopolysacharide activates CGRP-neurons in the parabrachial nucleus}

Intraperitoneal injection of LPS has been shown to activate CGRP ${ }^{P B N}$ neurons ${ }^{4}$. We examined if a low dose $(10 \mu \mathrm{g} / \mathrm{kg})$ of LPS activated CGRPPBN neurons in our experimental setting using immunofluorescent detection of the activity marker c-fos. As expected, LPS induced strong activation of neurons in the external lateral part of the PBN (fig 2A-C). Closer examination using dual labeling of GFP and c-fos in animals expressing eGFP under the Calca-promotor revealed an extensive colocalization showing that many of the activated neurons were expressing CGRP (fig. 2D-E). We next analyzed if the activation of the PBN was blunted in aCGRP-KO mice but found no significant difference in c-fos expression between aCGRP-KO mice and their $\mathrm{HZ}$ littermates (fig. 2F). 
We next examined a number of responses related to threat-induced regulation of food-intake in aCGRP-KO mice. As CGRPPBN neurons have been shown to be important for the reduction of food intake induced by inflammation ${ }^{4}$, we assessed if CGRP is important for limitation of food intake under inflammatory conditions. aCGRP-KO mice developed normal inflammation-induced anorexia, measured by consumption of standard chow 3 and $6 \mathrm{~h}$ after an ip. injection of LPS (10 $\mu \mathrm{g} / \mathrm{kg}$; fig. 3A). CGRP $^{\text {PBN }}$ neurons are also important for conditioned taste avoidance, i.e. the ability to avoid taste stimuli after their association with nausea or malaise ${ }^{16}$. We exposed animals to a novel taste $(0,15 \%$ saccharin solution) and administered LPS (10 $\mu \mathrm{g} / \mathrm{kg})$ afterwards. Both aCGRP-KO mice and their WT littermates reduced their saccharin solution intake after the pairing of the sweet taste with inflammation-induced malaise two days earlier (fig. 3B). The WT and aCGRP-KO mice also displayed the same degree of neophobia to the new taste as they drank similar amounts of saccharine solution at the first presentation (fig. $3 \mathrm{C}$ ). CGRP $^{\mathrm{PBN}}$ neurons are also important for the prevention of overeating $^{7}$, as measured by meal size. We tested if $\alpha$ CGRP-KO mice with ad libitum access to normal chow displayed an increased intake of palatable food when given access to it for a limited period $(2 h)$. The consumption of a sweet, chocolate-flavored drink was not affected by loss of aCGRP (fig. 3D).

Calca deletion does not influence nociceptive behaviors or the affective component of pain

To examine the role of CGRP in pain-related responses, we first injected diluted formalin into the paw of $\alpha$ CGRP-KO mice and their WT littermates and monitored their response to the inflammatory pain for 1 h. aCGRP-KO mice showed normal nociceptive responses (fig. 2e). aCGRP-KO mice also showed a similar reduction in locomotion as WT-mice after intraperitoneal injection of $0.6 \%$ acetic acid (fig 4BD). To assess the affective component of pain, we used conditioned place aversion. This is a wellestablished model for the affective component of pain and measures the avoidance of an environment in which the mice have experienced pain. Both WT and aCGRP-KO mice learned to avoid a chamber where they were subjected to thermal pain (floor with temperature set to $45^{\circ} \mathrm{C}$ ) (fig. $4 \mathrm{E}$ ). $\alpha \mathrm{CGRP}-\mathrm{KO}$ tended to stay longer on the hot floor in the heat escape test (fig $2 \mathrm{~F}$ ), but the difference did not reach statistical significance $(p=0.06)$.

\section{Discussion}

We demonstrate that $\alpha$ CGRP is dispensable for many behaviors mediated by CGRPPBN neurons. Inhibition of the CGRP ${ }^{P B N}$ neurons diminished LPS-induced anorexia ${ }^{4}$ and LPS-induced conditioned taste aversion ${ }^{16}$. We show that these behavioral responses are unaffected by the lack of aCGRP in the parabrachial-amygdaloid pathway. This finding contrasts to previous research showing an attenuated conditioned taste aversion in aCGRP-KO mice after LiCl-injection ${ }^{13}$. Further, our results indicate that 
Calca deletion does not lead to overeating of palatable food in sated mice nor excessive intake of a liquid with a novel taste, even if the CGRP ${ }^{P B N}$ neurons have been shown to be important for protection from overfeeding ${ }^{7}$ and for taste neophobia ${ }^{1}$. Along the same line, functional silencing of CGRP ${ }^{\mathrm{PBN}}$ neurons by tetanus toxin impairs the fear response associated with painful foot-shocks ${ }^{3}$ but we demonstrate that $\alpha$ CGRP-KO mice have normal affective responses to thermal pain, since they avoid the context associated with nociceptive stimulation. CGRP ${ }^{P B N}$ neurons have also been shown to mediate escape behavior (jumping) in the hot plate test ${ }^{3}$. We find that aCGRP KO-mice tend to stay longer on a hot floor in the heat escape test. However, the difference was not statistically significant $(P=0.06)$ even though many animals were tested, indicating that the possible role of CGRP is minor.

Our results indicate that even if Calca can be used as a marker for a specific subpopulation of parabrachial neurons encoding danger signals, the $\alpha$ CGRP itself is not necessary for most aspect of such encoding. Instead, it is likely that the release of glutamate by the same neurons ${ }^{17}$ is sufficient to mediate the responses studied. Similar findings have been reported for other neuropeptides. For example, deletion of Agouti-Related Protein has no ${ }^{18}$ or only minor ${ }^{19}$ effects on food-intake and energy homeostasis, which contrasts to the strong effects seen after activation, inactivation ${ }^{20,21}$ or ablation of the hypothalamic neurons expressing this neuropeptide ${ }^{22,23}$. Even if we found no effect of $\alpha$ CGRP deletion in our experiments, we cannot exclude the possibility that CGRPa in PBN neurons is involved in danger-related signaling in more chronic settings or in other behavioral responses.

In addition to being expressed in the PBN, $\alpha$ CGRP is found in a large population of primary afferents ${ }^{24}$. Several of the behavioral responses tested in this study are dependent on primary afferents, but our findings indicate that $\alpha$ CGRP in these neurons is dispensable for the pain-related responses studied. Our results contrast to a previous study suggesting aCGRP in TRPV1-expressing primary afferents to be important for visceral nociceptive signaling ${ }^{24}$.

It is very unlikely that the lack of changes in the behavior of the mutant mice can be attributed to the residual amounts of CGRP detected in the PBN. The weak CGRP-IR in the PBN can be explained by low expression of a second CGRP isoform, CGRP $\beta$, encoded by a separate gene, Calcb $b^{25}$. Since $\alpha$ CGRP and $\beta C G R P$ differ only by three amino acids in mice ${ }^{26}$, they are very difficult to distinguish with antibodybased detection methods. Co-expression of Calca and Calcb has been reported in different cranial nerve nuclei ${ }^{25}$. Nevertheless, no CGRP-IR was detected in the projections to the CeA in our mice lacking Calca, which indicates that CGRP signaling in this structure was absent or reduced to negligible levels. Since the mouse line used has been shown to have deficits in CGRP-dependent responses including hot flushes ${ }^{27}$ it is unlikely that compensatory mechanisms can explain the lack of effect in our studies. It is also unlikely that the co-occurring deletion of calcitonin, also encoded by $\mathrm{Calca}^{28}$, would mask any 
effect on behaviors related to nociception and food consumption, since calcitonin is mostly important for calcium homeostasis ${ }^{29}$.

In summary, we demonstrate that $\alpha \mathrm{CGRP}$ is dispensable for aversion and for danger-related behaviors mediated by Calca-expressing neurons of the parabrachial nucleus. This finding suggests that inhibition of CGRP ${ }^{\mathrm{PBN}}$ neurons is a more promising approach for anti-aversive treatment compared to interventions specifically targeting CGRP signaling.

\section{References}

1. Campos, C. A., Bowen, A. J., Roman, C. W. \& Palmiter, R. D. Encoding of danger by parabrachial CGRP neurons. Nature 555, 617-620 (2018).

2. Palmiter, R. D. The Parabrachial Nucleus: CGRP Neurons Function as a General Alarm. Trends in Neurosciences vol. 41 280-293 (2018).

3. Han, S., Soleiman, M., Soden, M., Zweifel, L. \& Palmiter, R. D. Elucidating an Affective Pain Circuit that Creates a Threat Memory. Cell 162, 363-374 (2015).

4. Carter, M. E., Soden, M. E., Zweifel, L. S. \& Palmiter, R. D. Genetic identification of a neural circuit that suppresses appetite. Nature 503, 111-114 (2013).

5. Paues, J., Engblom, D., Mackerlova, L., Ericsson-Dahlstrand, A. \& Blomqvist, A. Feedingrelated immune responsive brain stem neurons: Association with CGRP. Neuroreport 12, 2399-2403 (2001).

6. Campos, C. A. et al. Cancer-induced anorexia and malaise are mediated by CGRP neurons in the parabrachial nucleus. Nat. Neurosci. 2o, 934-942 (2017).

7. Campos, C. A., Bowen, A. J., Schwartz, M. W. \& Palmiter, R. D. Parabrachial CGRP Neurons Control Meal Termination. Cell Metab. 23, 811-820 (2016).

8. D’Hanis, W., Linke, R. \& Yilmazer-Hanke, D. M. Topography of thalamic and parabrachial Calcitonin Gene-Related Peptide (CGRP) immunoreactive neurons projecting to subnuclei of the amygdala and extended amygdala. J. Comp. Neurol. 505, 268-291 (2007).

9. Bowen, A. J. et al. Dissociable control of unconditioned responses and associative fear learning by parabrachial cgrp neurons. Elife 9, 1-50 (2020).

10. Han, J. S., Adwanikar, H., Li, Z., Ji, G. \& Neugebauer, V. Facilitation of synaptic transmission and pain responses by CGRP in the amygdala of normal rats. Mol. Pain 6, (2010).

11. Xu, W., Lundeberg, T., Wang, Y. T., Li, Y. \& Yu, L. C. Antinociceptive effect of calcitonin generelated peptide in the central nucleus of amygdala: Activating opioid receptors through amygdala-periaqueductal gray pathway. Neuroscience 118, 1015-1022 (2003). 
12. Han, J. S., Li, W. \& Neugebauer, V. Critical role of calcitonin gene-related peptide 1 receptors in the amygdala in synaptic plasticity and pain behavior. J. Neurosci. 25, 10717-10728 (2005).

13. Chen, J. Y., Campos, C. A., Jarvie, B. C. \& Palmiter, R. D. Parabrachial CGRP Neurons Establish and Sustain Aversive Taste Memories. Neuron 10o, 891-899.e5 (2018).

14. McCoy, E. S., Taylor-Blake, B. \& Zylka, M. J. CGRPa-expressing sensory neurons respond to stimuli that evoke sensations of pain and itch. PLoS One 7, (2012).

15. Schindelin, J. et al. Fiji: An open-source platform for biological-image analysis. Nature Methods vol. 9 676-682 (2012).

16. Carter, M. E., Han, S. \& Palmiter, R. D. Parabrachial calcitonin gene-related peptide neurons mediate conditioned taste aversion. J. Neurosci. 35, 4582-4586 (2015).

17. Alhadeff, A. L. et al. Excitatory hindbrain-forebrain communication is required for cisplatininduced anorexia and weight loss. J. Neurosci. 37, 362-370 (2017).

18. Qian, S. et al. Neither Agouti-Related Protein nor Neuropeptide Y Is Critically Required for the Regulation of Energy Homeostasis in Mice. Mol. Cell. Biol. 22, 5027-5035 (2002).

19. Wortley, K. E. et al. Agouti-related protein-deficient mice display an age-related lean phenotype. Cell Metab. 2, 421-427 (2005).

20. Aponte, Y., Atasoy, D. \& Sternson, S. M. AGRP neurons are sufficient to orchestrate feeding behavior rapidly and without training. Nat. Neurosci. 14, 351-355 (2011).

21. Krashes, M. J. et al. Rapid, reversible activation of AgRP neurons drives feeding behavior in mice. J. Clin. Invest. 121, 1424-1428 (2011).

22. Gropp, E. et al. Agouti-related peptide-expressing neurons are mandatory for feeding. Nat. Neurosci. 8, 1289-1291 (2005).

23. Luquet, S., Perez, F. A., Hnasko, T. S. \& Palmiter, R. D. NPY/AgRP neurons are essentials for feeding in adult mice but can be ablated in neonates. Science (80-. ). 310, 683-685 (2005).

24. Spencer, N. J. et al. CGRPa within the Trpv1-cre population contributes to visceral nociception. Am. J. Physiol. - Gastrointest. Liver Physiol. 314, G188-G200 (2018).

25. Amara, S. G. et al. Expression in brain of a messenger RNA encoding a novel neuropeptide homologous to calcitonin gene-related peptide. Science (80-. ). 229, 1094-1097 (1985).

26. Thomas, P. M., Nasonkin, I., Zhang, H., Gagel, R. F. \& Cote, G. J. Structure of the mouse calcitonin/calcitonin gene-related peptide alpha and beta genes. Mitochondrial DNA 12, 131135 (2001).

27. Wilhelms, D. B. et al. CGRP is critical for hot flushes in ovariectomized mice. Front. Pharmacol. 9, (2019). 
28. Amara, S. G., Jonas, V., Rosenfeld, M. G., Ong, E. S. \& Evans, R. M. Alternative RNA processing in calcitonin gene expression generates mRNAs encoding different polypeptide products. Nature 298, 240-244 (1982).

29. Davey, R. A. \& Findlay, D. M. Calcitonin: Physiology or fantasy? Journal of Bone and Mineral Research vol. 28 973-979 (2013).

\section{Acknowledgements}

This work was supported by the Swedish Medical Research Council, the Knut and Alice Wallenberg foundation, the Swedish Brain foundation, the Linköping Parkinson Foundation and the County Council of Östergötland. We thank Redoy Ullah, Julia Levinsson and Susanne Bauer for contributions to the experimental work.

\section{Author contributions}

JZ, JS and DE planned the study and designed the experiments. JZ and JS did the majority of the experimental work and AKS and MJ provided help and assistance. MJ was responsible for genotyping. JZ, JS and DE prepared a draft of the manuscript that all authors read and commented.

\section{Competing interests}

The authors declare no competing interests.

\section{Figure legends}

Figure 1. CGRP levels are strongly attenuated in aCGRP-KO mice. A-F) Micrographs from confocal microscopy showing that the CGRP-immunoreactivity in the parabrachial nucleus was strongly reduced in the aCGRP-KO mice compared to heterozygous $(\mathrm{Hz})$ mice. Scale bar 50: $\mu \mathrm{m}$. G-L) CGRPimmunoreactivity was present in the central nucleus of the amygdala of $\mathrm{Hz}$, but not aCGRP-KO mice. Scale bar: $100 \mu \mathrm{m}$. PBN - parabrachial nucleus, CeA - central amygdala, BIA - basolateral amygdala. 
Figure 2. C-fos expression in the PBN after LPS-injection. A-B) Representative fluorescent images showing c-fos immunoreactivity in the PBN of one $\mathrm{NaCl}(\mathrm{A})$ and one LPS (B) injected WT animal. Scale bar $50 \mu \mathrm{m}$. C) The amount of C-fos positive cells in the PBN is higher $3 \mathrm{~h}$ after LPS injection i.p. compared to $3 \mathrm{~h}$ after $\mathrm{NaCl}$ injection in WT animals. (T-test: $p=0.02, \mathrm{NaCl} n=4$, LPS $n=4)$. D-E) Representative confocal images of the PBN showing c-fos expression (red) in CGRP-neurons expressing GFP (green) from one $\mathrm{Hz}(\mathrm{D})$ and one $\mathrm{KO}(\mathrm{E})$ animal. Scale bar $10 \mu \mathrm{m}$. F) Loss of CGRP does not affect the number of c-fos expressing cells in the PBN after LPS injection. ( $\mathrm{Hz} / \mathrm{NaCl} n=4, \mathrm{~Hz} / \mathrm{LPS} n=4, K O / L P S$ $n=4)$

Figure 3. Food consumption in aCGRP-KO mice. A) Inflammation-induced anorexia was normal in the aCGRP-KO animals. Two-way RM ANOVA followed by Holm-Sidak's multiple comparisons test: interaction: $F_{(3,20)}=8.969, p=0.0006$; time: $F_{(1,20)}=125.3 ; p<0.001 ;$ treatment: $F_{(3,20)}=17.48 ; p<$ $0,001 .{ }^{*} p<0.01 * * * p<0.001 * * * * p<0.0001 .{ }^{*}$ indicate comparisons within the same genotype and time after injection; $n=6$ in both groups. B) Mice without $\alpha$ CGRP develop normal conditioned taste avoidance. One-way ANOVA followed by Holm-Sidak's multiple comparisons test: $F_{(2,27)}=9.605, p=$ 0.0007. ${ }^{* *} p<0.01, \mathrm{NaCl} n=8 \mathrm{WT}+7 \alpha \mathrm{CGRP}-\mathrm{KO}, \mathrm{WT} n=11, \alpha \mathrm{CGRP-KO} n=8$. C) $\alpha \mathrm{CGRP}-\mathrm{KO}$ mice show normal taste neophobia. Two-way ANOVA followed by Holm-Sidak's multiple comparisons test: interaction: $F_{(1,53)}=0.5417, p=0.4650$; time: $F_{(1,53)}=8.3 ; p=0.0057$; genotype: $F_{(1,53)}=0.02358 ; p=$ 0.8785. WT $1^{\text {st }} n=20$, WT $2^{\text {nd }} n=10, \alpha C G R P-K O 1^{\text {st }} n=20, \alpha C G R P-K O 2^{\text {nd }} n=7$. D) Palatable food intake was unaffected by deletion of Calca. T-test: $p=0.1733$; WT $n=11$, aCGRP-KO $n=10$.

Figure 4. Nociceptive behaviors in aCGRP-KO mice. A) $\alpha$ CGRP-KO mice showed a normal response to inflammatory pain in the formalin test. One-way RM ANOVA: time: $F_{(11,120)}=6.52, p<0.0001$; genotype: $F_{(1,120)}=0.351, p=0.5548$; interaction: $F_{(11,120)}=0.638, p=0.7927 ; n=6$ in both groups. B) Conditioned place aversion to thermal pain was normal in $\alpha$ CGRP-KO mice. T-test: $p=0.7865$; WT $n=$ 5, aCGRP-KO $n=4$. C) No statistically significant difference in escape latency was seen between the 
genotypes in the heat escape test. T-test: $\mathrm{p}=0.06$; WT $n=17$; $\alpha$ CGRP-KO $n=19$. D) Timeline of the acetic acid locomotion test. E) No genotype difference was seen in locomotor activity after intraperitoneal injection of saline or $0.6 \%$ acetic acid. F) $\alpha$ CGRP-KO and WT mice show similar reduction in locomotion following acetic acid injection compared to after saline injection. WT $n=9 ; \alpha$ CGRP-KO $n=11$. 


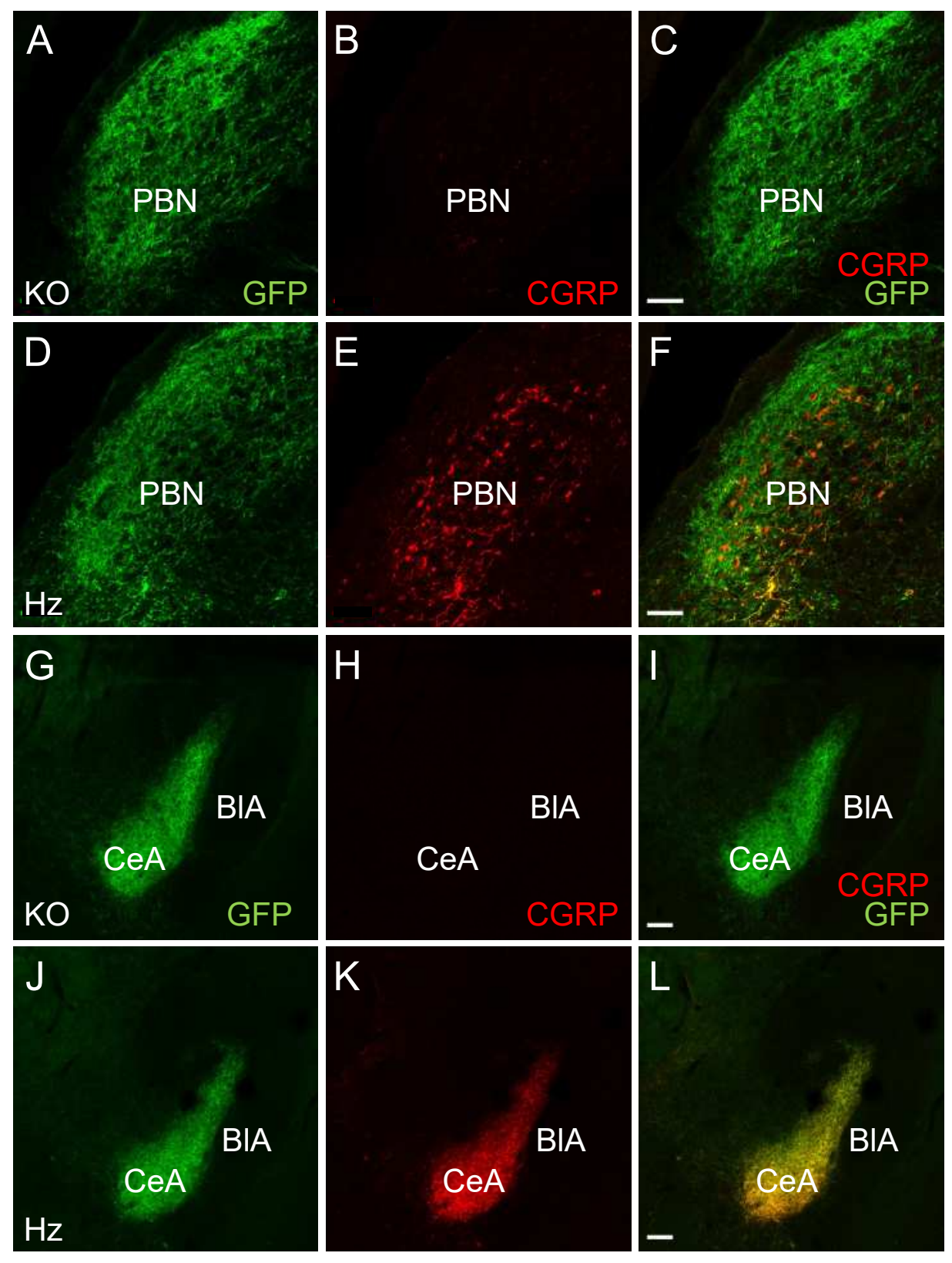

Figure 1. CGRP levels are strongly attenuated in aCGRP-KO mice.

A-F) Micrographs from confocal microscopy showing that the CGRP-immunoreactivity in the parabrachial nucleus was strongly reduced in the aCGRP-KO mice compared to heterozygous (Hz) mice. Scale bar 50: $\mu \mathrm{m}$. G-L) CGRP-immunoreactivity was present in the central nucleus of the amygdala of $\mathrm{Hz}$, but not aCGRP-KO mice. Scale bar: 100 $\mu \mathrm{m}$. PBN - parabrachial nucleus, CeA - central amygdala, BIA - basolateral amygdala. 

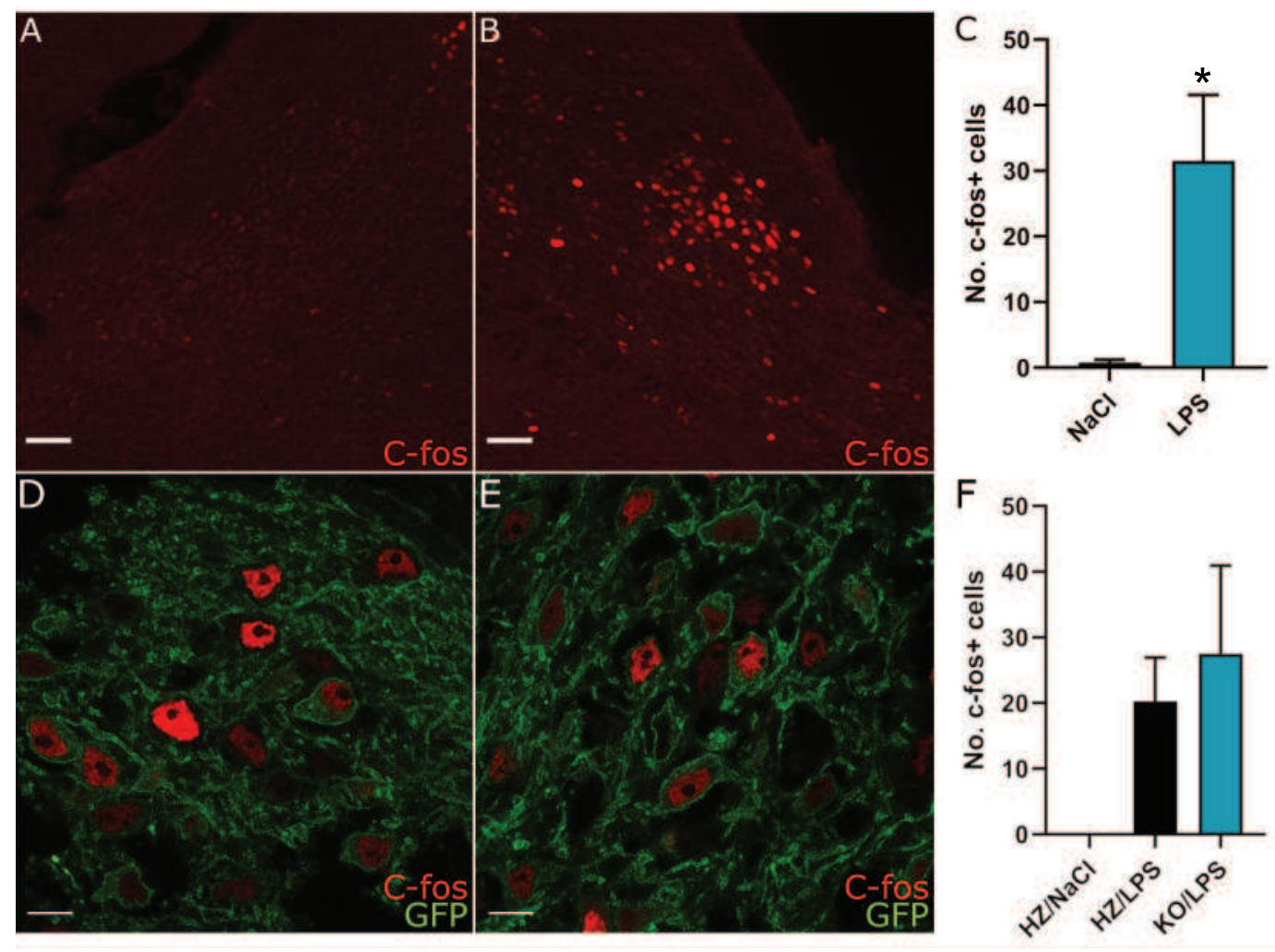

Figure 2. C-fos expression in the PBN after LPS-injection.

A-B) Representative fluorescent images showing c-fos immunoreactivity in the PBN of one $\mathrm{NaCl}(\mathrm{A})$ and one LPS (B) injected WT animal. Scale bar $50 \mu \mathrm{m}$. C) The amount of $\mathrm{C}$-fos positive cells in the PBN is higher $3 \mathrm{~h}$ after LPS injection i.p. compared to $3 \mathrm{~h}$ after $\mathrm{NaCl}$ injection in WT animals. (T-test: $p=0.02, \mathrm{NaCl} n=4, \mathrm{LPS} n=4$ ). D-E) Representative confocal images of the PBN showing c-fos expression (red) in CGRP-neurons expressing GFP (green) from one $\mathrm{Hz}(\mathrm{D})$ and one $\mathrm{KO}(\mathrm{E})$ animal. Scale bar $10 \mu \mathrm{m}$. F) Loss of CGRP does not affect the number of c-fos expressing cells in the PBN after LPS injection. $(\mathrm{Hz} / \mathrm{NaCl} \mathrm{n}=4, \mathrm{~Hz} / \mathrm{LPS} \mathrm{n}=4, \mathrm{KO} / \mathrm{LPS} \mathrm{n}=4)$ 
A
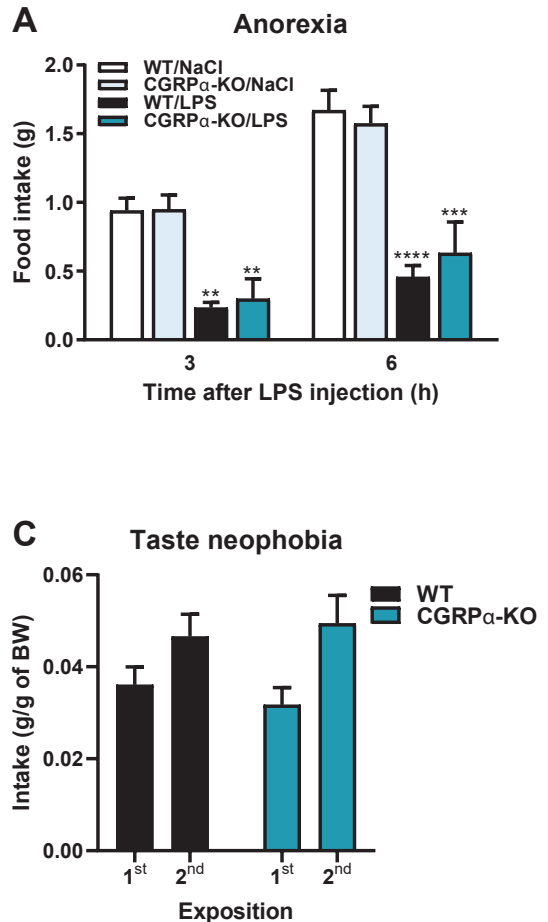

B

CTA
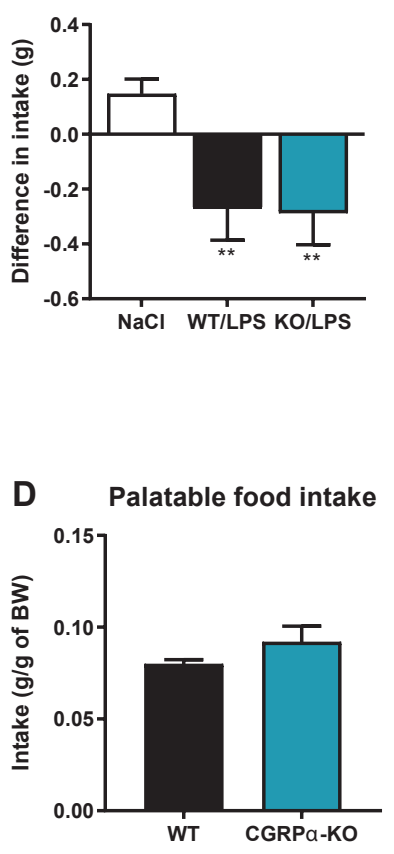

Figure 3. Food consumption in aCGRP-KO mice.

A) Inflammation-induced anorexia was normal in the aCGRP-KO animals. Two-way RM ANOVA followed by Holm-Sidak's multiple comparisons test: interaction: $F(3,20)=8.969$, $p$ $=0.0006$; time: $F(1,20)=125.3 ; p<0.001$; treatment: $F(3,20)=17.48 ; p<0,001 .{ }^{* *} p<0.01$ ${ }^{* * *} p<0.001{ }^{* * * *} p<0.0001 .{ }^{*}$ indicate comparisons within the same genotype and time after injection; $n=6$ in both groups. B) Mice without aCGRP develop normal conditioned taste avoidance. One-way ANOVA followed by Holm-Sidak's multiple comparisons test: $F(2,27)=$ 9.605, $p=0.0007 .{ }^{* *} p<0.01, \mathrm{NaCl} n=8 \mathrm{WT}+7$ aCGRP-KO, WT $n=11$, aCGRP-KO $n=$ 8. C) aCGRP-KO mice show normal taste neophobia. Two-way ANOVA followed by Holm-Sidak's multiple comparisons test: interaction: $F(1,53)=0.5417, p=0.4650$; time: $F(1,53)=8.3 ; p=0.0057$; genotype: $F(1,53)=0.02358 ; p=0.8785$. WT 1 st $n=20$, WT 2nd $n=10$, aCGRP-KO 1st $n=20$, aCGRP-KO 2nd $n=7$. D) Palatable food intake was unaffected by deletion of Calca. T-test: $p=0.1733$; WT $n=11$, $\alpha$ CGRP-KO $n=10$. 

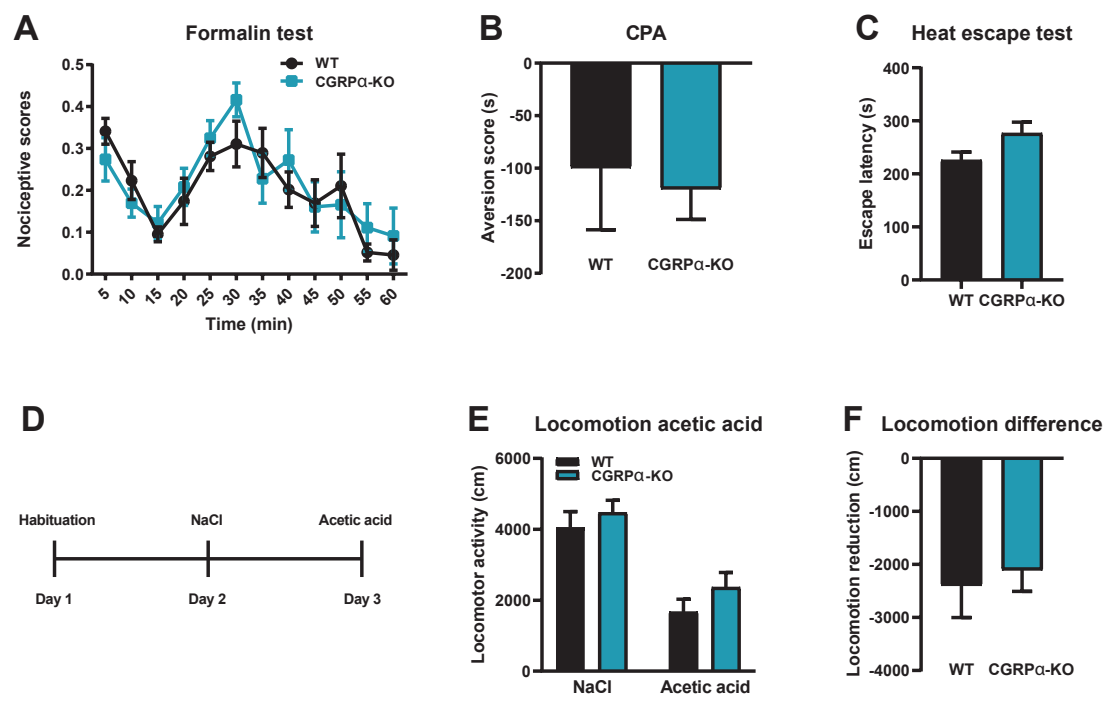

Figure 4. Nociceptive behaviors in aCGRP-KO mice.

A) aCGRP-KO mice showed a normal response to inflammatory pain in the formalin test. One-way RM ANOVA: time: $F(11,120)=6.52, p<0.0001$; genotype: $F(1,120)=0.351, p$ $=0.5548$; interaction: $F(11,120)=0.638, p=0.7927 ; n=6$ in both groups. $B$ ) Conditioned place aversion to thermal pain was normal in aCGRP-KO mice. T-test: $p=0.7865$; WT $n$ $=5$, $\mathrm{aCGRP}-\mathrm{KO} n=4$. C) No statistically significant difference in escape latency was seen between the genotypes in the heat escape test. T-test: $p=0.06$; WT $n=17 ; \alpha$ CGRP-KO $n=19$. D) Timeline of the acetic acid locomotion test. E) No genotype difference was seen in locomotor activity after intraperitoneal injection of saline or $0.6 \%$ acetic acid. F) aCGRP-KO and WT mice show similar reduction in locomotion following acetic acid injection compared to after saline injection. WT $n=9$; $\alpha$ CGRP-KO $n=11$. 
Figures

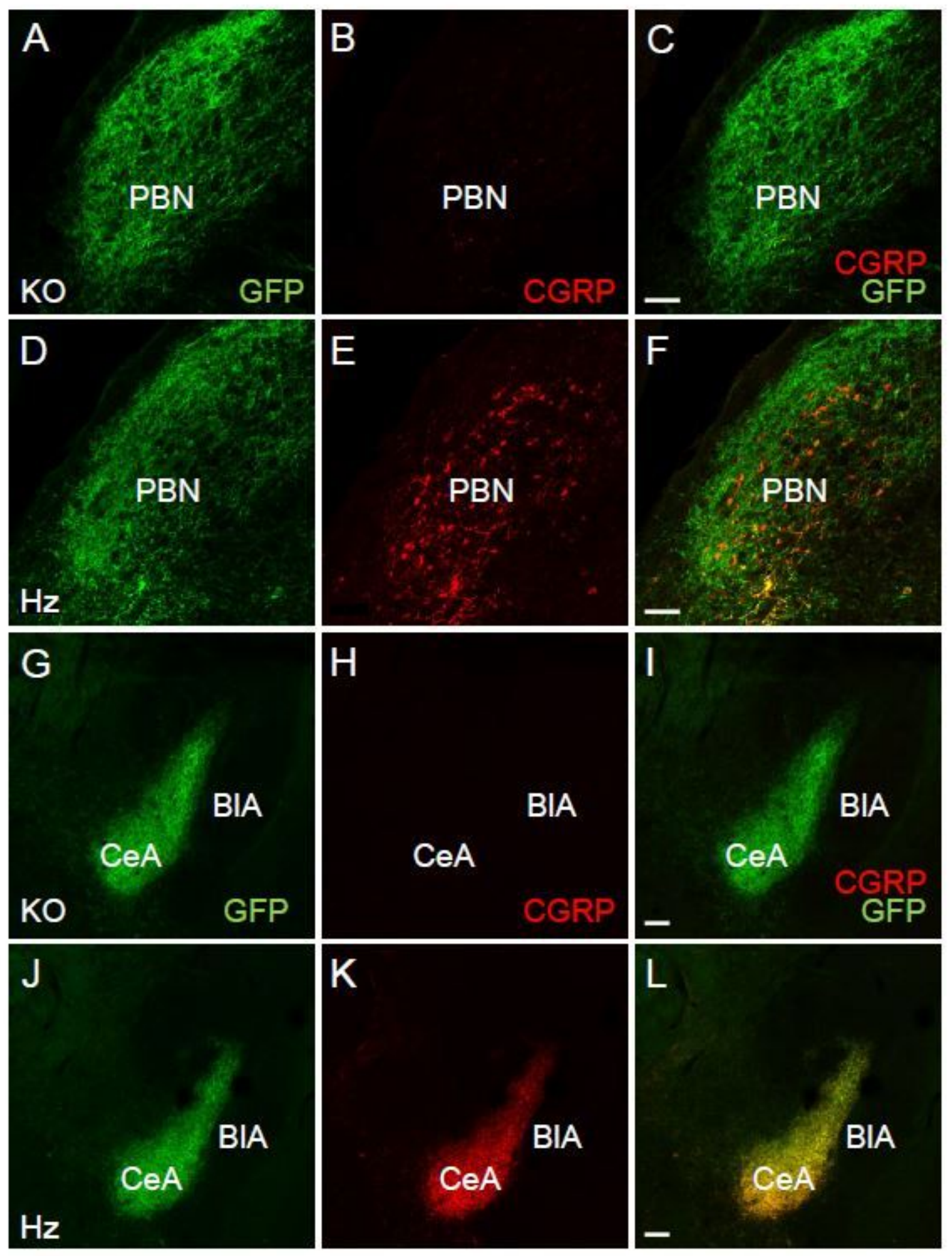

Figure 1

CGRP levels are strongly attenuated in aCGRP-KO mice. A-F) Micrographs from confocal microscopy showing that the CGRP-immunoreactivity in the parabrachial nucleus was strongly reduced in the aCGRPKO mice compared to heterozygous $(\mathrm{Hz})$ mice. Scale bar 50: $\mu \mathrm{m}$. G-L) CGRP-immunoreactivity was 
present in the central nucleus of the amygdala of Hz, but not aCGRP-KO mice. Scale bar: $100 \mu \mathrm{m}$. PBN parabrachial nucleus, $\mathrm{CeA}$ - central amygdala, BIA - basolateral amygdala.
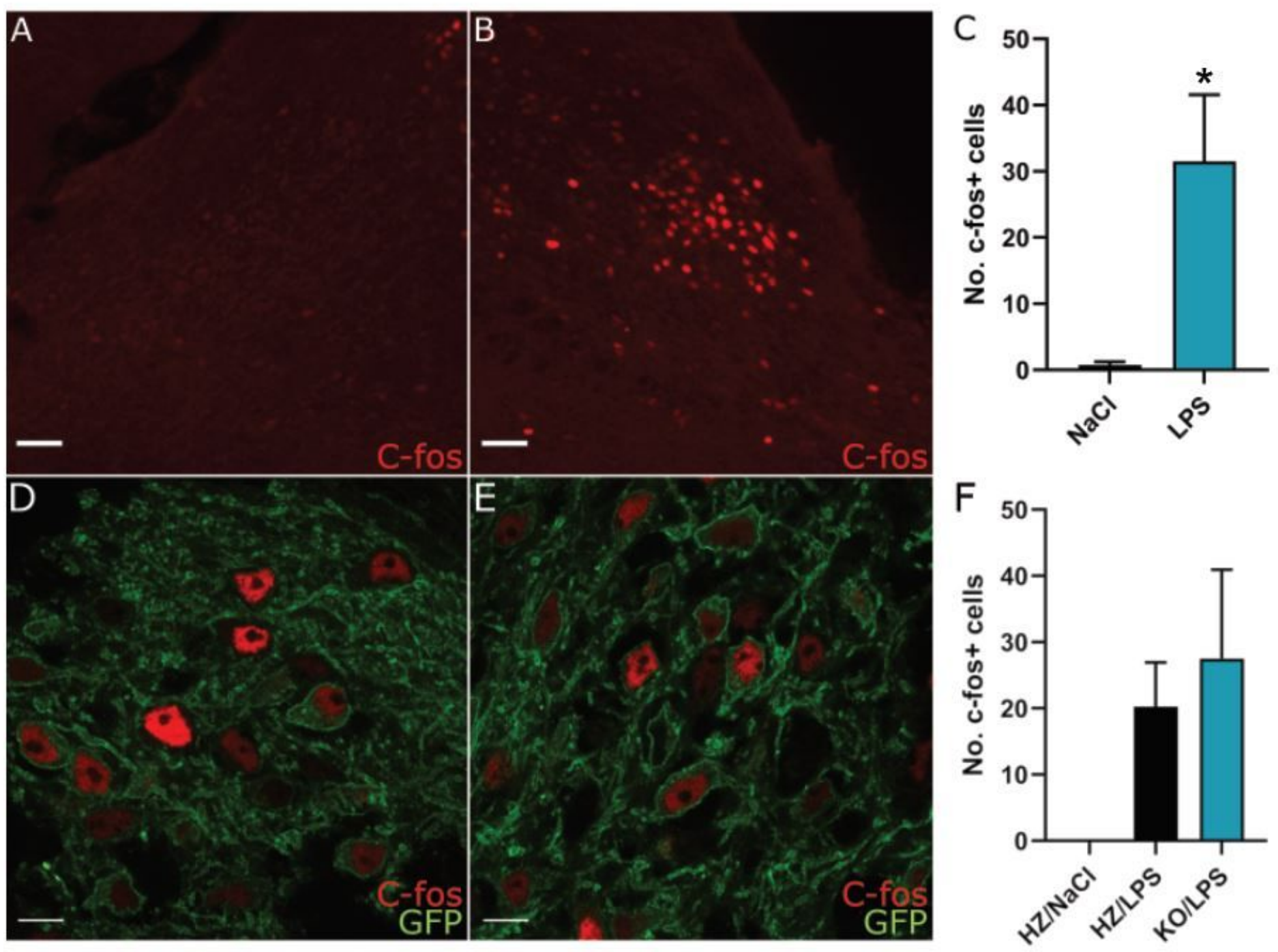

Figure 2

C-fos expression in the PBN after LPS-injection. A-B) Representative fluorescent images showing c-fos immunoreactivity in the PBN of one $\mathrm{NaCl}(\mathrm{A})$ and one LPS (B) injected WT animal. Scale bar $50 \mu \mathrm{m} . \mathrm{C}$ ) The amount of $\mathrm{C}$-fos positive cells in the PBN is higher $3 \mathrm{~h}$ after LPS injection i.p. compared to $3 \mathrm{~h}$ after $\mathrm{NaCl}$ injection in WT animals. (T-test: $p=0.02, \mathrm{NaCl} n=4$, LPS $n=4$ ). D-E) Representative confocal images of the PBN showing c-fos expression (red) in CGRP-neurons expressing GFP (green) from one $\mathrm{Hz}(\mathrm{D})$ and one $\mathrm{KO}(\mathrm{E})$ animal. Scale bar $10 \mu \mathrm{m}$. F) Loss of CGRP does not affect the number of c-fos expressing cells in the PBN after LPS injection. $(\mathrm{Hz} / \mathrm{NaCl} n=4, \mathrm{~Hz} / \mathrm{LPS} n=4, K O / L P S \mathrm{n}=4)$ 
A

Anorexia

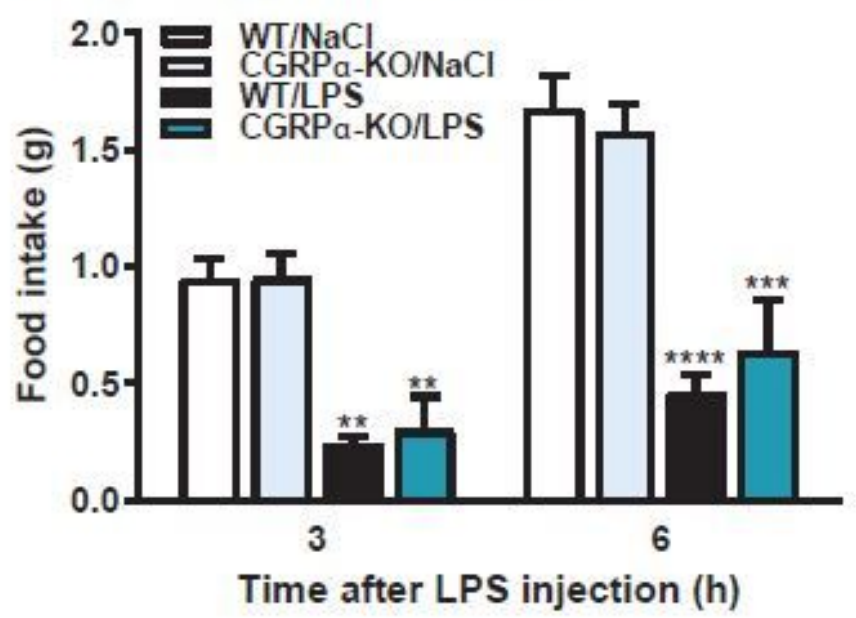

Time after LPS injection (h)

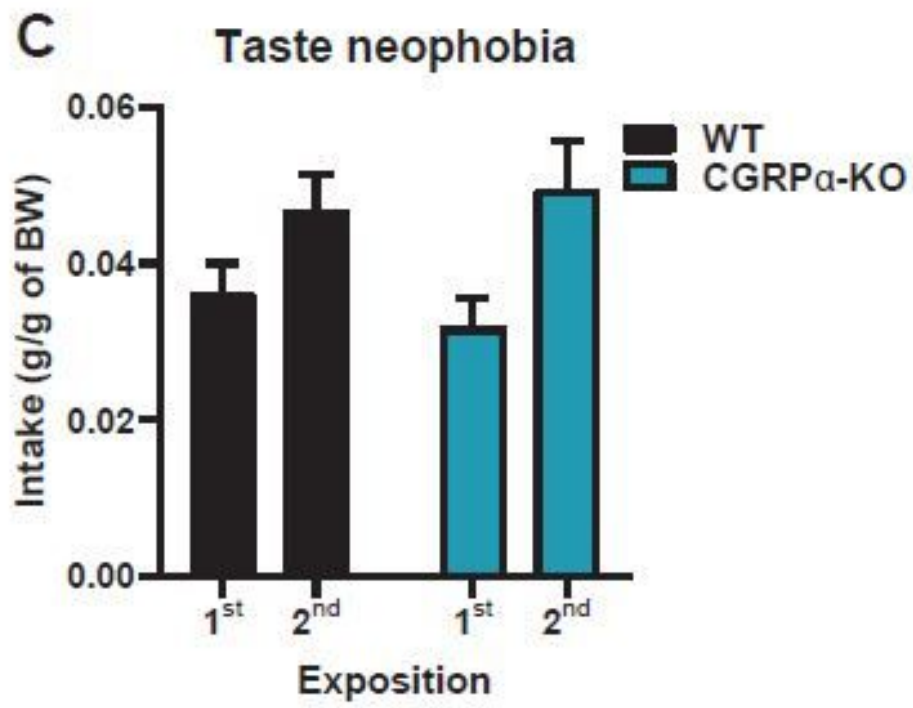

B CTA

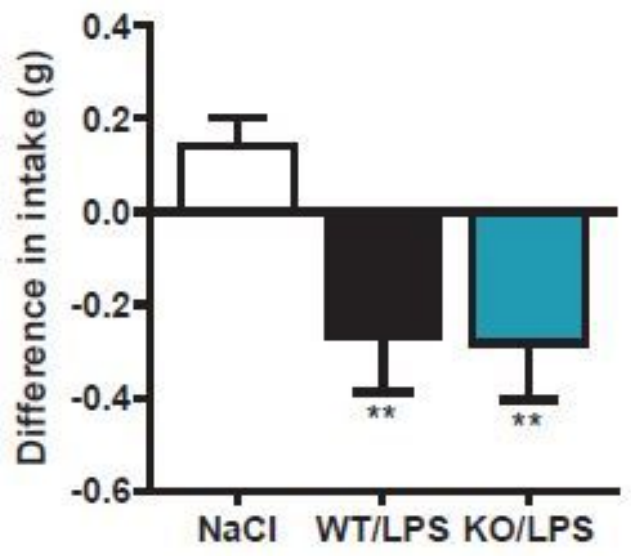

D Palatable food intake

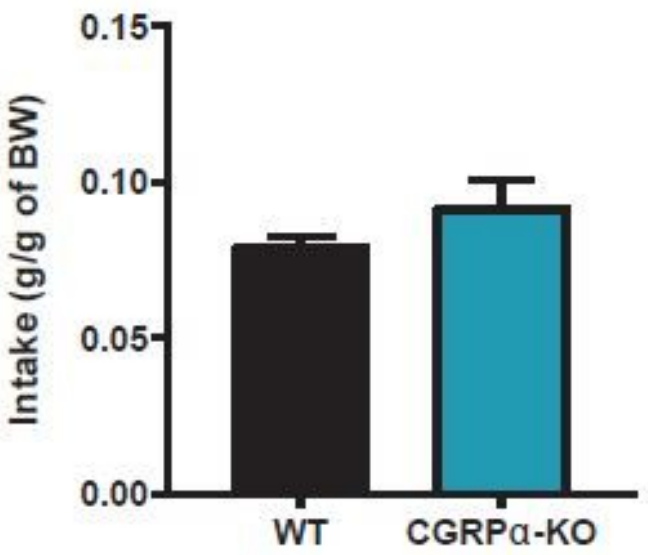

Figure 3

Food consumption in aCGRP-KO mice. A) Inflammation-induced anorexia was normal in the aCGRP-KO animals. Two-way RM ANOVA followed by Holm-Sidak's multiple comparisons test: interaction: $F(3,20)=$ 8.969, $p=0.0006$; time: $F(1,20)=125.3 ; p<0.001$; treatment: $F(3,20)=17.48 ; p<0,001 .{ }^{* \star} p<0.01 * \star * p$ $<0.001 * \star \star \star p<<0.0001$. *indicate comparisons within the same genotype and time after injection; $\mathrm{n}=6$ in both groups. B) Mice without aCGRP develop normal conditioned taste avoidance. One-way ANOVA followed by Holm-Sidak's multiple comparisons test: $F(2,27)=9.605, p=0.0007 .{ }^{\star *} p<0.01, \mathrm{NaCl} n=8$ WT + 7 aCGRP-KO, WT $n=11$, aCGRP-KO $n=8$. C) aCGRP-KO mice show normal taste neophobia. Twoway ANOVA followed by Holm-Sidak's multiple comparisons test: interaction: $F(1,53)=0.5417, p=$ 0.4650; time: $F(1,53)=8.3 ; p=0.0057$; genotype: $F(1,53)=0.02358 ; p=0.8785$. WT 1 st $n=20$, WT $2 n d n$ 
$=10$, aCGRP-KO 1st $n=20$, aCGRP-KO 2nd $n=7$. D) Palatable food intake was unaffected by deletion of Calca. T-test: $p=0.1733 ;$ WT $n=11$, aCGRP-KO $n=10$.
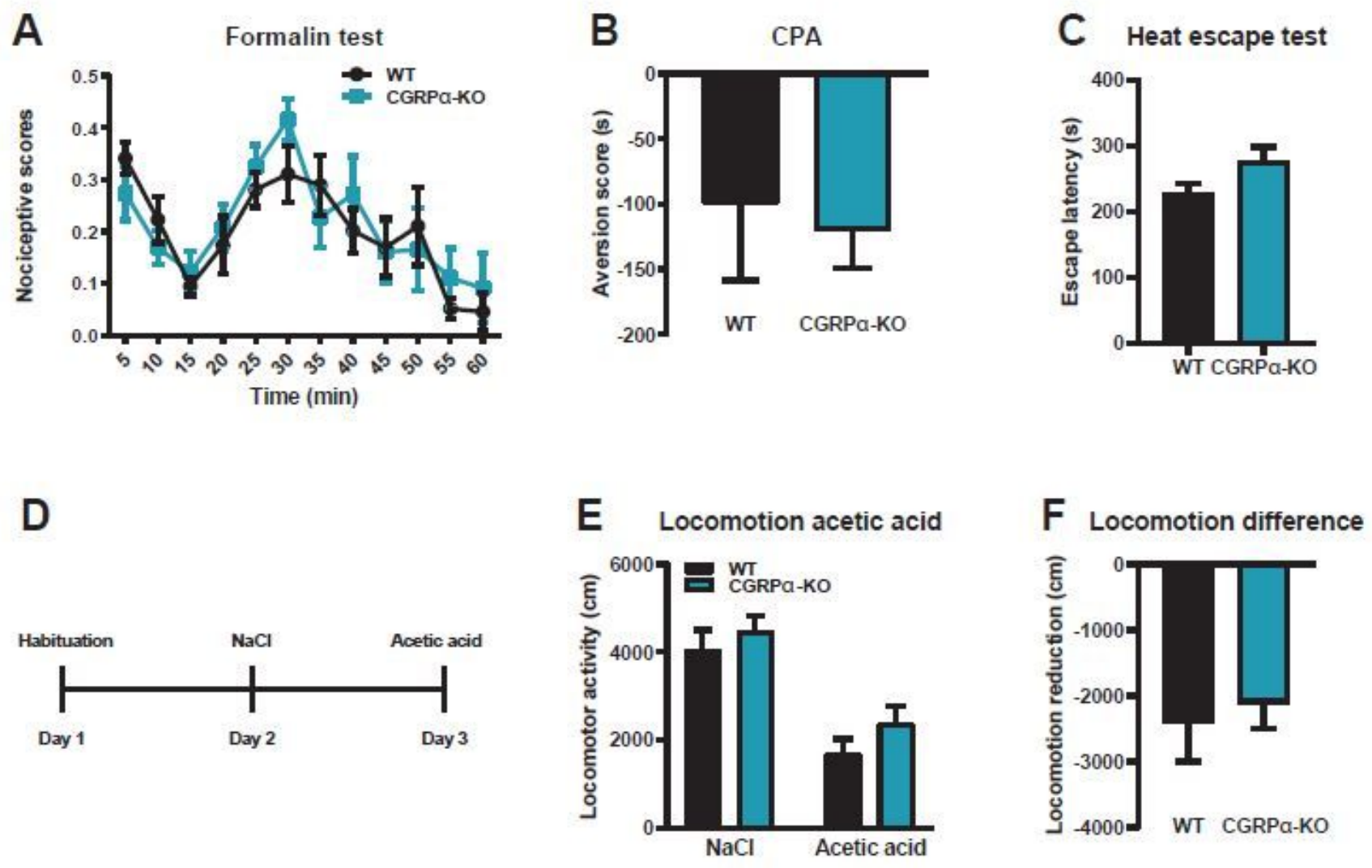

\section{Figure 4}

Nociceptive behaviors in aCGRP-KO mice. A) aCGRP-KO mice showed a normal response to inflammatory pain in the formalin test. One-way RM ANOVA: time: $F(11,120)=6.52$, $p<0.0001$; genotype: $F(1,120)=$ $0.351, p=0.5548$; interaction: $F(11,120)=0.638, p=0.7927 ; n=6$ in both groups. B) Conditioned place aversion to thermal pain was normal in aCGRP-KO mice. T-test: $p=0.7865$; WT $n=5$, aCGRP-KO $n=4 . C$ ) No statistically significant difference in escape latency was seen between the genotypes in the heat escape test. T-test: $p=0.06$; WT $n=17$; $a$ CGRP-KO $n=19$. D) Timeline of the acetic acid locomotion test. E) No genotype difference was seen in locomotor activity after intraperitoneal injection of saline or $0.6 \%$ acetic acid. F) aCGRP-KO and WT mice show similar reduction in locomotion following acetic acid injection compared to after saline injection. WT $n=9 ;$ aCGRP-KO $n=11$. 